\title{
Realistic 3D Computer Model of the Gerbil Middle Ear, Featuring Accurate Morphology of Bone and Soft Tissue Structures
}

\author{
Jan A. N. Buytaert ${ }^{1}$, Wasil H. M. Salih $^{1}$, Manual Dierick ${ }^{2}$, Patric Jacobs ${ }^{2}$, and Joris J. J. Dirckx ${ }^{1}$ \\ ${ }^{1}$ Laboratory of BioMedical Physics, University of Antwerp, Groenenborgerlaan 171, 2020 Antwerp, Belgium \\ ${ }^{2}$ Centre for X-ray Tomography, Ghent University, Proeftuinstraat 86, 9000 Gent, Belgium
}

Received: 28 January 2011; Accepted: 20 June 2011; Online publication: 13 July 2011

\begin{abstract}
In order to improve realism in middle ear (ME) finiteelement modeling (FEM), comprehensive and precise morphological data are needed. To date, micro-scale $\mathrm{X}$-ray computed tomography $(\mu \mathrm{CT})$ recordings have been used as geometric input data for FEM models of the ME ossicles. Previously, attempts were made to obtain these data on ME soft tissue structures as well. However, due to low X-ray absorption of soft tissue, quality of these images is limited. Another popular approach is using histological sections as data for 3D models, delivering high in-plane resolution for the sections, but the technique is destructive in nature and registration of the sections is difficult. We combine data from high-resolution $\mu \mathrm{CT}$ recordings with data from high-resolution orthogonal-plane fluorescence opticalsectioning microscopy (OPFOS), both obtained on the same gerbil specimen. State-of-the-art $\mu \mathrm{CT}$ delivers high-resolution data on the 3D shape of ossicles and other ME bony structures, while the OPFOS setup generates data of unprecedented quality both on bone and soft tissue ME structures. Each of these techniques is tomographic and non-destructive and delivers sets of automatically aligned virtual sections. The datasets coming from different techniques need to be registered with respect to each other. By combining both datasets, we obtain a complete high-resolution morphological model of all functional components in the gerbil ME. The resulting 3D model can be readily imported in FEM software and is made freely available to the research
\end{abstract}

Correspondence to: Jan A. N. Buytaert - Laboratory of BioMedical Physics - University of Antwerp - Groenenborgerlaan 171, 2020 Antwerp, Belgium. Telephone: +32-3-2653553; fax: +32-3-2653318; email: jan.buytaert@ua.ac.be community. In this paper, we discuss the methods used, present the resulting merged model, and discuss the morphological properties of the soft tissue structures, such as muscles and ligaments.

Keywords: gerbil, middle ear, modeling, high resolution, three-dimensional, soft tissue, surface mesh, micro-scale X-ray computed tomography (micro-CT), orthogonal-plane fluorescence opticalsectioning microscopy (OPFOS)

\section{INTRODUCTION}

The middle ear (ME) forms a small 3D biomechanical system. It mainly consists of the tympanic membrane (TM), three ossicles - malleus, incus, and stapes - and their supporting ligaments and muscles. The remarkable performance of ME mechanics is too complex to be understood intuitively. For better understanding, ME modeling was introduced. Finite-element (computer) modeling (FEM) has become an established numerical technique to simulate ME mechanics. In ME research, the technique was first introduced by Funnell and Laszlo (1978). As one of its inputs, FEM requires 3D morphological computer models of the ME components. These mesh models consist of a finite number of elements, e.g., tetrahedra or hexahedra.

Current morphological models are either incomplete, low resolution, and/or contain rudimentary shapes to represent (some) ME components. Pioneering work in this field used manually drawn geometrical shapes in the computer to represent the ME malleus, incus, and stapes (Wada et al. 1992; Ladak and Funnell 
1996; Blayney et al. 1997; Eiber et al. 2000; Prendergast et al. 2000; Koike et al. 2002). Some authors used low- or modest-resolution shapes measured with medical X-ray computed tomography (CT) (Rodt et al. 2002; Lee et al. 2006) or with tabletop micro-CT $(\mu \mathrm{CT})$ devices (Decraemer et al. 2002, 2003; Elkhouri et al. 2006; Puria and Steele 2010; Lee et al. 2010). Other authors used histological sectioning (Funnell et al. 1992; Sun et al. 2002) or magnetic resonance microscopy (MRM, NMR, MRI) (Funnell et al. 2005; Elkhouri et al. 2006), but again with modest resolutions. In many models, the suspensory ligaments and muscle tendons are either omitted (Wada et al. 1992; Ladak and Funnell 1996; Blayney et al. 1997; Lord et al. 1999; Rodt et al. 2002) or manually incorporated as simple geometrical objects such as blocks, cylinders, or cones (Prendergast et al. 2000; Beer et al. 2000; Koike et al. 2002; Sun et al. 2002; Lee et al. 2006). To the authors' knowledge, only models by Wang et al. (2006), Gan et al. (2007), and Cheng and Gan (2008) (using histological sectioning) and by Mikhael et al. (2004), Sim and Puria (2008), and Ruf et al. 2009 (using X-ray techniques) contain actually measured shapes of soft tissue structures, but in low resolution.

To improve realism in FEM calculations, ME geometry models need to incorporate all and accurate shapes of the ossicles and suspensory soft tissue structures (Decraemer et al. 2003). As the computer calculating capacity has grown to a point where it can manage large amounts of data and as the scientific measurement apparatus is now capable of high-resolution imaging on all kinds of tissue types, the time has come to incorporate realistic and complete morphological 3D ME models in FEM. We point out that it might not be necessary, even not numerically feasible, to perform FEM with all structures described in the highest detail. On the other hand, it is difficult to decide beforehand how precise the morphologic model needs to be. Therefore, we think it is important to first have a high-resolution morphologic model available, which can then be simplified to the modeler's judgment.

In the current paper, we provide these high-quality models by combining data originating from two different tomographic techniques: State-of-the-art $\mu \mathrm{CT}$ tomography allows to obtain precise data on bony structures, but due to the low X-ray absorption of soft tissue, CT generates poor quality images of soft tissue (Lemmerling et al. 1997). Therefore, we combine these data with measurements from another and relatively new technique: orthogonal-plane fluorescence optical-sectioning (OPFOS) microscopy or tomography. This method images both bone and soft tissue at the same time and in high resolution. As gerbil is one of the standard laboratory animal models in fundamental hearing research, we chose this species for our first model.

\section{MATERIALS AND METHODS}

\section{Dissection}

All animal manipulations in this work were performed in accordance with Belgian legislation and the directives set by the Ethical committee on Animal Experimentation of our institution (University of Antwerp, Belgium). Three adult Mongolian gerbils (Meriones unguiculatus), aged between 3 and 6 months, were used. They were housed in cages with food and water ad libitum in our animal facility.

The animals were euthanized using carbon dioxide, followed by a cardiac perfusion with physiological fluid to rinse out all the blood from the gerbil head blood vessels. This step is necessary to allow for OPFOS tomography (as we will explain below). The gerbils are then decapitated and the right temporal bones were isolated. The specimens were reduced in size until only the bulla was left containing the middle and inner ear, cf. Figure 1. During the harvesting of these bullas, continuous moistening with mist from an ultrasonic humidifier (Bionaire BT204) was applied to avoid dehydration.

\section{Cross-sectional imaging of bone with X-ray tomography}

The first stage of 3D tomographic recording of the ME was achieved using micro-scale X-ray computed tomography. The dissected bullae were enclosed in separate Eppendorf vials, together with a calibration object and a few droplets of physiological fluid at the bottom. In this way, a $100 \%$ saturated humid environment was created to avoid dehydration artifacts. Another droplet of fluid was placed in the ear canal-which could help-to distinguish the outline border of the TM shape with the air-filled ME cavity. Water and air have a slightly different X-ray absorption coefficient, so a layer of water on the

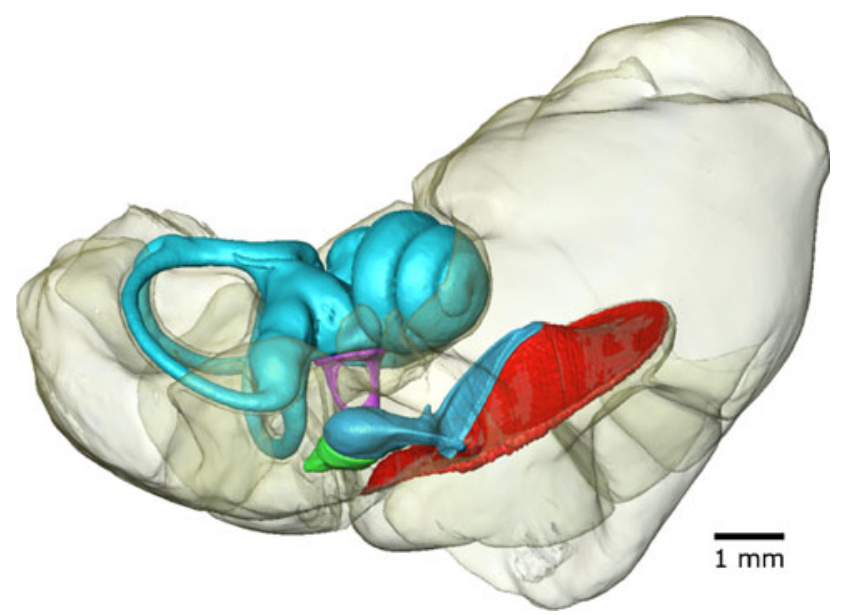

FIG. 1. 3D model of separate surface meshes of bony middle and inner ear components of gerbil 2, obtained from $\mu \mathrm{CT}$. The bulla is rendered transparent. Voxel size is $8.5 \times 8.5 \times 8.5 \mu \mathrm{m}$. 
extremely thin TM can help to reveal its medial shape outline. In previous work, we measured the shape of the eardrum before and after putting fluid on the membrane: Even with a $10 \mathrm{~mm}$ water column in the ear canal, no measurable deformation was found with moiré profilometry of $15 \mu \mathrm{m}$ resolution (Buytaert et al. 2009). As the droplet of water is less than $3 \mathrm{~mm}$ high (inducing a pressure load of $30 \mathrm{~Pa}$ ), the TM deformation is well below the $\mu \mathrm{CT}$ measurement resolution. The Eppendorf vials (made from polypropylene) are almost X-ray transparent. Especially bone absorbs X-rays well, thus creating a high contrast in transmission recordings. The small calibration objects were custom-made from polyvinyl chloride in our mechanical workshop and possess about the same X-ray absorption properties as thin bone (Gea et al. 2005). They served as an independent calibration to verify the $\mu \mathrm{CT}$ device specifications.

The vials containing gerbil specimens were scanned at the UGCT scanning facility at Ghent University (www.ugct.ugent.be) using a custom-built $\mu \mathrm{CT}$ scanner of medium energy (up to $160 \mathrm{keV}$ ). The scanner has a directional X-ray tube with a feature recognition capability up to $2 \mu \mathrm{m}$ (Masschaele et al. 2007). The scans were performed at a tube voltage of $120 \mathrm{kV}$ (photon energy levels ranging from 0 to $120 \mathrm{keV}$ ) and a current of $58 \mu \mathrm{A}$. A custom-made vial holder was mounted on a computer-controlled rotation table (MICOS, UPR160F-AIR). For each specimen, a series of 1,000 shadow projections of $1,496 \times 1,880$ pixels was recorded covering an object rotation of $360^{\circ}$ (or one recording every $0.36^{\circ}$ ). Reconstruction of the tomographic data volume to serial sections was achieved using the back-projection algorithms of the Octopus software package (Dierick et al. 2004), resulting in 1,780 reconstructed cross sections of $1,496 \times 1,496$ pixels. From these calculated cross sections with an isometric pixel size of $8.5 \mu \mathrm{m}$, accurate 3D models of the three ossicles and other bony structures were generated. All three datasets cover a volume of $15.1 \times 12.7 \times 12.7 \mathrm{~mm}$ $(1,780 \times 1,496 \times 1,496 \times 8.5 \mu \mathrm{m})$.

Cross-sectional imaging of soft tissue with optical tomography

Due to the low X-ray absorption of soft tissue, another tomographic technique was needed: OPFOS microscopy (Voie et al. 1993). OPFOS was initially developed to image the inner ear cochlea, but it has also been used in ME studies (Voie 2002; Buytaert and Dirckx 2007, 2009). In the OPFOS method, parallel optical sections through a macroscopic biomedical specimen are created by means of a thin sheet of laser light, and the fluorescence originating from within the cross section of the light sheet with the tissue is recorded in the direction perpendicular to the plane of the laser light. The light emitted by the specimen originates from autofluorescence or from staining the specimen with a fluorescent dye. OPFOS images both bone and soft tissue at the same time and in real time, as no (back-projection) calculations are required. It allows region-of-interest (ROI) imaging and has both a high sectioning and a high in-plane resolution. Hence, perfectly and automatically aligned images of virtual cross sections can be obtained. OPFOS scanning was performed at the Laboratory of BioMedical Physics at the University of Antwerp (www.ua.ac.be/bimef) with a custom-built setup using bi-directional light-sheet illumination (Buytaert and Dirckx 2009; Buytaert 2010).

For OPFOS imaging, an elaborate specimen preparation is needed (Voie 2002; Buytaert and Dirckx 2009), as the technique requires the specimens to be perfectly transparent. Before $\mu \mathrm{CT}$ scanning, all blood was removed from the blood vessels, as coagulated blood cannot be made transparent afterward. After $\mu \mathrm{CT}$ recording, a $10 \%$ neutral buffered formalin bath was applied. Next, all calcium was removed using $10 \%$ EDTA in water solution combined with microwaves. Because of this decalcification, the OPFOS method has to be performed second after $\mu$ CT X-ray scanning. Then, the specimens were dehydrated using a slowly graded ethanol series, up till $100 \%$. Next, all tissue was refractive index matched using a slowly graded Spalteholz fluid series, again up till $100 \%$. As a result, the specimens become entirely transparent when submerged in pure Spalteholz fluid. Finally, to obtain stronger fluorescence, the specimens are stained with rhodamine $\mathrm{B}$.

Both soft tissue and bone were made transparent and fluorescent; hence, both tissue types are visualized with the technique. We focused on ROI OPFOS imaging of ME ligaments, tendons, and muscles, while images of the (often larger) bony structures are more easily obtained from $\mu \mathrm{CT}$. Comparison of highresolution $\mu \mathrm{CT}$ and OPFOS data allows us to distinguish bone from soft tissue in the OPFOS data. Merging of the two datasets generates the complete ME model with all of its functional components accounted for.

The shape of the TM was obtained from the $\mu \mathrm{CT}$ data. The OPFOS technique is able to visualize this extremely thin tissue when performing ROI imaging on a small part of the membrane, cf. Figure 2. However, to image the membrane full-field with OPFOS, one needs to zoom out and the resolution needed to adequately visualize this thin membrane is lost. Furthermore, the eardrum is prone to preparation artifacts: Because the gerbil specimens went through an extensive procedure of tissue fixation, decalcification, dehydration, and Spalteholz treatment, the extremely thin TM can get deformed. Therefore, the data on eardrum shape are obtained 

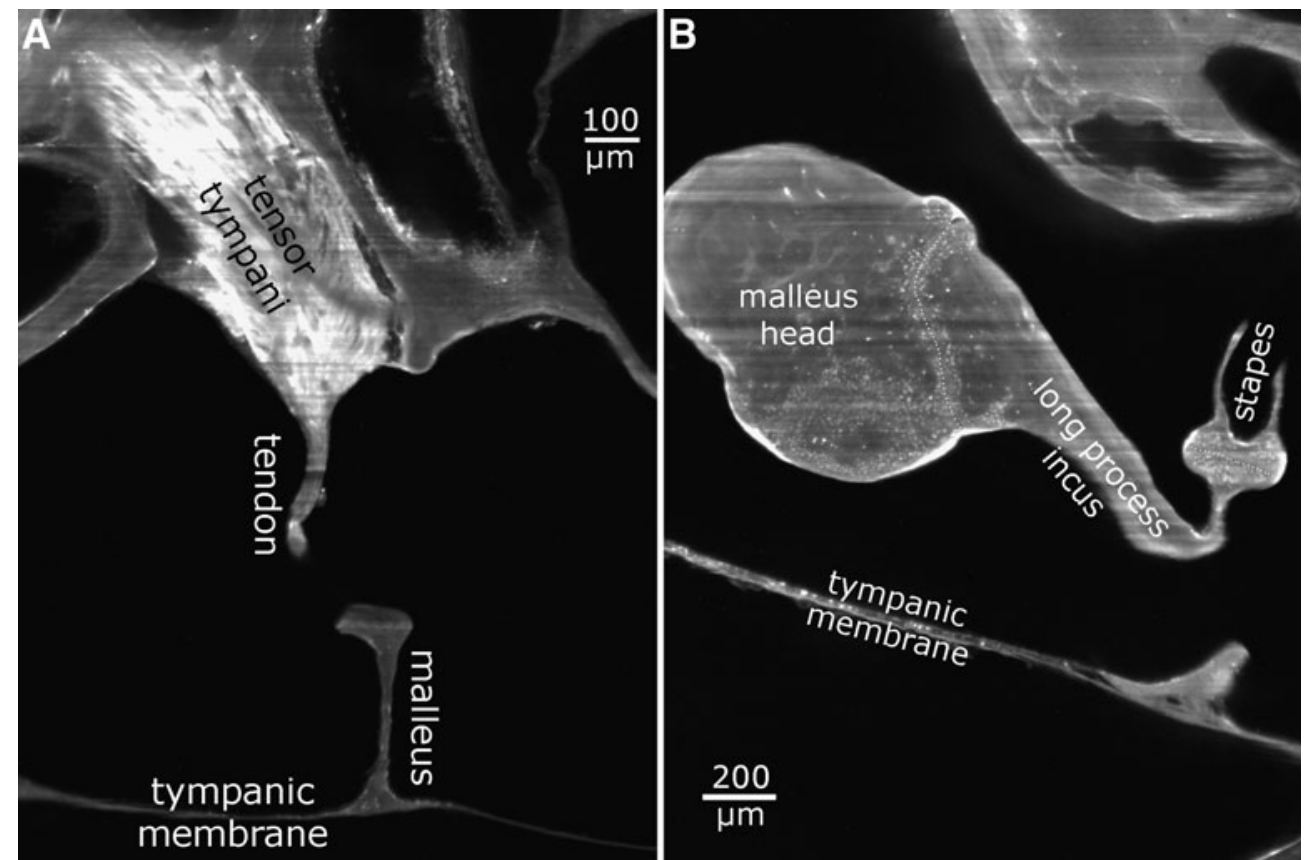

FIG. 2. 2D virtual cross sections delivered by the OPFOS technique. A Tensor tympani tendon reaching down toward malleus. B Incudomallear and incudostapedial articulation. Pixel size $1.5 \times 1.5 \mu \mathrm{m}$.

from the CT images, recorded before any specimen processing was applied. X-rays are normally not suited to image soft tissue, especially if it is very thin, like the eardrum. We tried to counter this problem by applying a droplet of physiological fluid through the ear canal on top of the membrane. The medial border of the droplet and eardrum then becomes more easily distinguishable from air in the ME cavity. In this way, the membrane outline will be obtained without deformation and with adequate resolution.

Apart from the specimen preparation, the OPFOS method has another disadvantage as it suffers from stripe artifacts. Opaque regions or areas of less transparency locally reduce the intensity of the laser light sectioning sheet, causing shadow lines or stripes in the rest of the image. This is partially countered by simultaneous dual light-sheet illumination in our setup (Buytaert and Dirckx 2009). Measuring and analyzing the OPFOS data is very time-consuming; therefore, only one gerbil ear has been processed. On the other hand, the $\mu \mathrm{CT}$ data of all three gerbils were analyzed.

\section{Visual observations}

We performed visual observations of the orientation, location, shape, and suspension of the ossicular chain inside opened ME bullae with an operating microscope (Zeiss, OPMI Sensera S7). When 3D computer data, models and results were obtained from $\mu \mathrm{CT}$ or OPFOS with striking features, they were compared to qualitative observations of the real geometry in opened bullae with the operating microscope to verify their interpretation. These experiences gave us the necessary expertise to confirm the 3D model results and conclusions of the present paper. For instance, after a targeted dissection, we could visually confirm that the posterior incudal ligament in gerbil indeed exists as one whole band instead of two separate structures, as we found in our OPFOS data and model.

\section{D segmentation and reconstruction}

After obtaining several series of object cross sectionsone $\mu \mathrm{CT}$ set originating from back-projection calculations and several ROI datasets from direct OPFOS recordings - we identified and segmented the relevant structures in all images. The goal of segmentation is to locate objects boundaries, which in turn allows software to build 3D surface meshes by triangulation.

In our case, segmentation was done manually for thousands of sections using the commercial image segmentation and 3D surface mesh generating software package Amira 5.3 (Visage Imaging). Manual segmentation might seem primitive and time-consuming, but using our morphological expertise, manual segmentation delivers better results than purely automated segmentation based on thresholding of gray scale values. The Amira software package uses the marching cubes algorithm for triangulation. It takes eight neighboring voxel locations at a time (forming an imaginary cube), after which the polygon(s) needed to represent the part of the isosurface that passes through this cube 
are determined. The individual polygons are finally fused into the intended surface. This leads to subvoxel triangulation that easily manages sharp angles. When smoothing or simplification (reduction of the number of triangles) is used, the program takes the "steepness" of the surface into account: Flat surface parts are more reduced than curved parts.

As final result, we end up with triangulated surface meshes for the $\mu \mathrm{CT}$ and OPFOS datasets. These can be further developed into finite-element volume meshes using Amira or other packages. On the website of the Laboratory of BioMedical Physics group, we suggest some powerful and open-source volume generating software, e.g., PreView.

\section{Merging of CT and OPFOS models}

All cross sections in a $\mu \mathrm{CT}$ dataset and therefore all models of ME components originating from it are inherently perfectly aligned within the data stack. The OPFOS datasets were focused on the soft tissue by separate ROI recordings. However, parts of the bone are included in the OPFOS ROI recordings as well. The cross sections within each ROI OPFOS data stack are also perfectly aligned, but the resulting mesh models per stack are unrelated to the other OPFOS datasets (because of different ROI zooming and/or other slicing orientation) and unrelated to the CT dataset and models.

To merge the OPFOS data with the $\mu \mathrm{CT}$ data, the $\mu \mathrm{CT}$ dataset was used as a reference. We did not merge the 2D image cross sections, but the 3D mesh models: All partial bone models from ROI OPFOS were three-dimensionally aligned to corresponding parts of the $\mu \mathrm{CT}$ models using an iterative spatial transformation least-squares minimization process of the Amira software package. This process uses the iterative closest point (ICP) algorithm to minimize the difference between two point clouds (e.g., all surface nodes of, respectively, an OPFOS and a $\mu \mathrm{CT}$ mesh model). ICP iteratively revises the spatial transformation (6 degrees of freedom for translation and rotation) needed to minimize the Euclidean distance between the points of two datasets. This concept is referred to as the Procrustes superimposition method: The root mean square (RMS) of the distances between corresponding points of the two surfaces is evaluated. Corresponding point pairs are created by finding the closest point of the reference $(\mu \mathrm{CT})$ surface mesh for each point of the other (OPFOS) surface mesh. When the two surfaces are identical and perfectly superimposed, the RMS of all corresponding point distances will be zero. In the case of the OPFOS versus the $\mu \mathrm{CT}$ stapes model for instance, we obtained a root mean square difference of $17 \mu \mathrm{m}$ (or two $\mu \mathrm{CT}$ voxels). After obtaining such a good match between the OPFOS and $\mu \mathrm{CT}$ bone model, we applied the same spatial transformation to the OPFOS soft tissue mesh(es) from that OPFOS dataset. In this way, all OPFOS datasets were combined with $\mu \mathrm{CT}$ data into one model.

\section{RESULTS}

\section{Computed tomography}

Three gerbil ears were recorded with $\mu \mathrm{CT}$, delivering three isometric data stacks of reconstructed cross sections (pixel size $8.5 \times 8.5 \mu \mathrm{m}$, separated $8.5 \mu \mathrm{m}$ ). To illustrate the image quality, we present one $\mu \mathrm{CT}$ cross section in Figure 3. Full movies of all cross sections are available on our website, and the entire dataset is available upon request. Notice how distinguishable the ossicle boundaries, the incudomallear and incudostapedial joint cleft, and the annular ligament cleft are in the figure. This high contrast and resolution facilitates the segmentation process considerably.

Our main attention went to the ME, but separate 3D surface meshes were also created of the fluid-filled bony labyrinth of the inner ear (cochlea scalae and modiolus, and vestibular apparatus), cf. Figures 1 and 4 . The ME bulla air cavities of all gerbils are modeled as well. They give an indication of the enclosed air volume in the ME, cf. Table 1. These segmented volumes include the volume of the ossicles, ligaments, and muscles. Finally, a separate rudimentary mesh of all bone using a fixed segmentation threshold was made. Using transparent rendering for this large model, one can virtually look inside the bulla and observe the ossicles and inner ear inside, cf. Figure 1. We listed volume, dimensions, and several other properties of the ossicles, the TM and the ME bulla cavity in Tables 1, 2, and 3. These and other quantitative data are readily and accurately available from our models.

The mass of malleus, incus, and stapes are, respectively, $1.145,0.633$, and $0.116 \mathrm{mg}$ as reported by Nummela (1995). Adopting these representative values for our specimen in combination with the volumes given in Table 1, we get an average ossicle bone density of $1.37 \times 10^{3} \mathrm{~kg} / \mathrm{m}^{3}$ for the stapes and $1.74 \times 10^{3} \mathrm{~kg} / \mathrm{m}^{3}$ for incus and malleus.

Note that the outline of the TM was surprisingly but successfully visualized using $\mu \mathrm{CT}$. The resolution was just high enough to show the shape outline of the extremely thin membrane. Thickness information could not be obtained. Using a fluid droplet in the ear canal to aid in distinguishing the medial border of the eardrum partially failed, as can be seen in Figure 3, fluid is not covering the entire membrane surface in the ear canal because of an air bubble. 


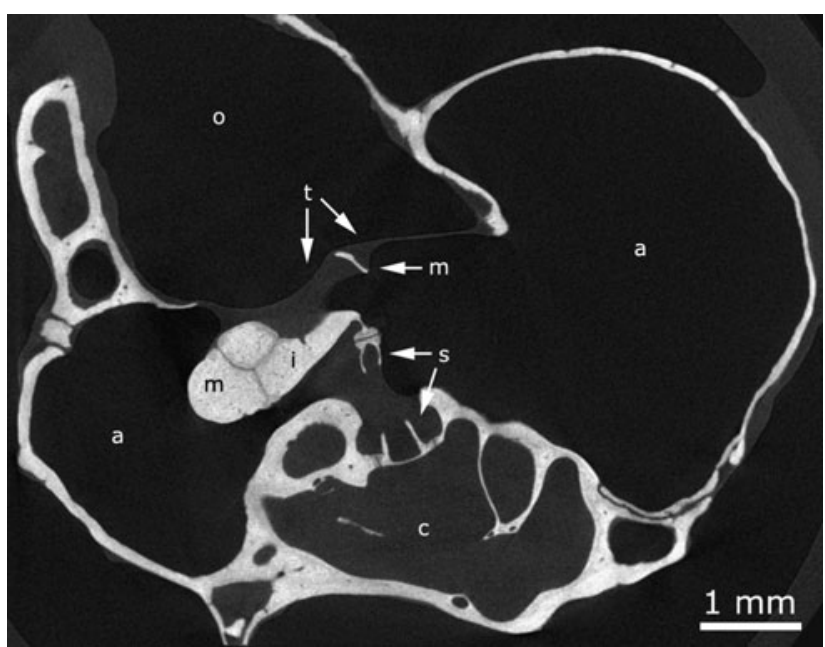

FIG. 3. Reconstructed $\mu \mathrm{CT}$ cross section through gerbil 1 (originally $1,496 \times 1,496$ pixels cropped to $740 \times 950$ pixels). a middle ear air cavity, $c$ inner ear cochlea, $i$ incus, $m$ malleus, $o$ outer ear canal, $s$ stapes, $t$ tympanic membrane outline. Pixel size is $8.5 \times 8.5 \mu \mathrm{m}$.

Finally, we could observe channels (blood vessels) inside the ossicles, occurring especially in the incus and malleus bone, cf. Figure 5. The ossicular surface shapes are almost identical between all three animals, and the same is true for the size, volume, and branching layout of the major channels inside them.

\section{OPFOS tomography}

We will now discuss all identified (soft) tissue structures of the ME of gerbil 2, measured with OPFOS.

Posterior incudal ligament. Using $\mu \mathrm{CT}$, the posterior incudal ligament cannot be found, cf. Figure 6A, B, while using OPFOS it is clearly visible, cf. Figure $6 \mathrm{C}-\mathrm{E}$. This comparison between the two tomographic imaging techniques clearly demonstrates the usefulness of combining the two methods.

After segmentation and 3D representation, cf. Figure 6F, G, one can see that the ligament is built as one whole part and forms one sickle-shaped band of fibrous tissue. Its tiny volume amounts to $0.013 \mathrm{~mm}^{3}$. The sickle has its smallest thickness (orthogonally to the image plane of Fig. 6F) of $42 \mu \mathrm{m}$ near the incus short process and broadens to $190 \mu \mathrm{m}$ toward the bulla edge. The contact area at the middle ear cavity wall is also a bit larger than the contact area on the incus crus.

Anterior mallear ligament. The anterior process of the malleus has the shape of a (partially opened) handheld Japanese folding fan, reaching toward the anterior bulla wall, cf. Figures 7 and 8 . The connective soft tissue of the anterior mallear ligament, which should connect the process to the bulla, is undistinguishable from bone, both in the OPFOS and in the CT recordings. This ligament is probably more ossified or cartilaginous than in some other species, and no separate soft tissue model could be made.

Superior mallear and incudal ligament and lateral mallear ligament. According to real-time OPFOS observations, no superior mallear and incudal ligament are present in the gerbil $\mathrm{ME}$, which is confirmed by visual observations with the operating microscope. In addition, no lateral mallear ligament could be discerned with either method.

Tensor tympani muscle and tendon. Figure 2A shows a high-resolution OPFOS section image through the tensor tympani muscle and tendon, the TM, the malleus' manubrium, and the bulla. This image demonstrates OPFOS' capability to image bone and soft tissue in high resolution.

After the segmentation and triangulation process, the volume of the tensor tympani muscle and tendon can be calculated from the obtained 3D model and was found to be $0.486 \mathrm{~mm}^{3}$. The distance between the two most distant points on the combined structure is $3.25 \mathrm{~mm}$. The diameter of the muscle tendon varies between 50 and $80 \mu \mathrm{m}$.

The cross-sectional area of a muscle (rather than volume or length) determines the amount of force it
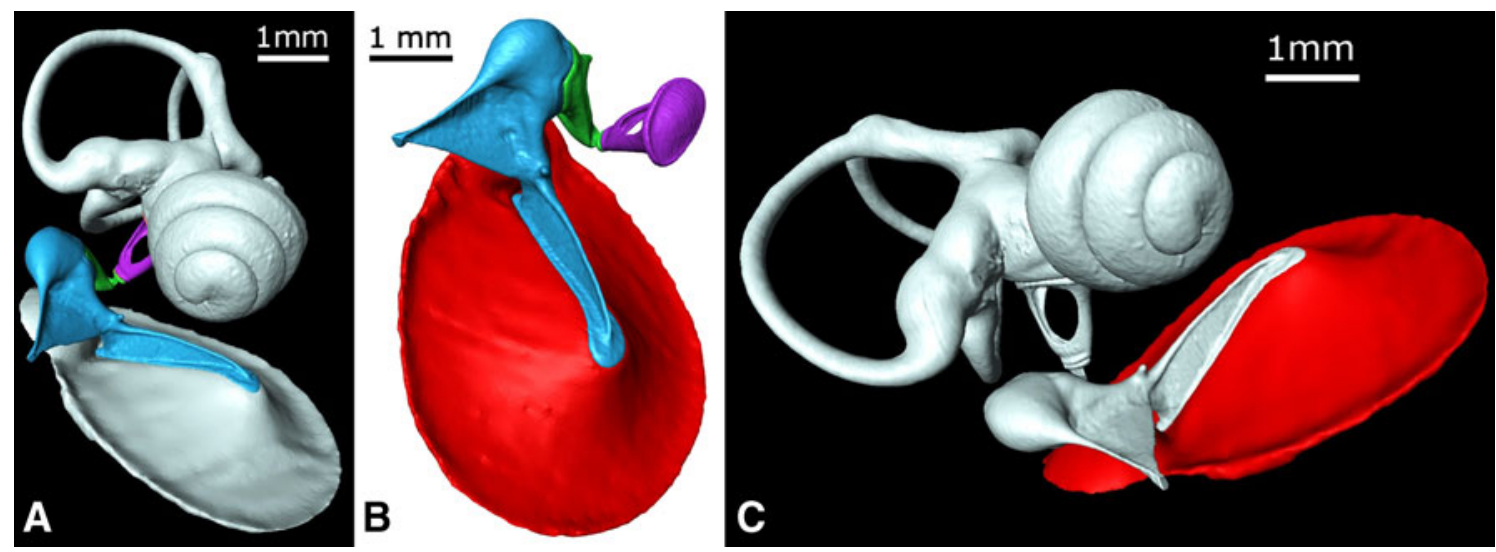

FIG. 4. 3D surface meshes. Voxel size is $8.5 \times 8.5 \times 8.5 \mu \mathrm{m}$. A Tympanic membrane+middle ear ossicles+inner ear fluid (gerbil 1). B Tympanic membrane+ middle ear ossicles (gerbil 2). C Tympanic membrane+middle ear ossicles+inner ear fluid (gerbil 3). 


\section{TABLE 1}

Volume, surface area, and number of triangles for G1, G2, and G3 ear components, derived from the 3D surface meshes obtained from $\mu \mathrm{CT}$

\begin{tabular}{lrrr}
\hline & $\begin{array}{l}\text { Volume } \\
\left(\mathrm{mm}^{3}\right)\end{array}$ & Area $\left(\mathrm{mm}^{2}\right)$ & Triangles (\#) \\
\hline Malleus G1 & 0.670 & 9.25 & 384,346 \\
Malleus G2 & 0.655 & 9.43 & 422,420 \\
Malleus G3 & 0.656 & 9.16 & 380,570 \\
Incus G1 & 0.374 & 3.85 & 160,772 \\
Incus G2 & 0.373 & 3.85 & 172,254 \\
Incus G3 & 0.344 & 3.54 & 148,328 \\
Stapes G1 & 0.085 & 3.88 & 159,118 \\
Stapes G2 & 0.082 & 3.88 & 175,242 \\
Stapes G3 & 0.082 & 3.65 & 149,374 \\
Middle ear cavity G1 & 221.680 & 386.50 & $4,999,382$ \\
Middle ear cavity G2 & 238.158 & 419.69 & $2,478,224$ \\
Middle ear cavity G3 & 239.542 & 412.96 & $2,999,806$ \\
Inner ear cavity G1 & 10.378 & 65.90 & $2,729,618$ \\
Inner ear cavity G2 & 10.439 & 69.14 & $2,998,432$ \\
Inner ear cavity G3 & 10.789 & 64.00 & $2,654,126$ \\
\hline
\end{tabular}

The ME cavity volume incorporates the air, ossicles, and ME soft tissue volume

G1 gerbil 1, G2 gerbil 2, G3 gerbil 3

can generate. A first rough estimate of the order of magnitude of the maximum generated force of the muscle can be derived as follows: By dividing the muscle belly volume by an average muscle fiber length of $400 \mu \mathrm{m}$ (estimated from the OPFOS images), we end up with a cross-sectional area of $1.2 \times 10^{-2} \mathrm{~cm}^{2}$. A common conversion factor from this area to the maximal isometric contraction force is given by $25 \mathrm{~N} / \mathrm{cm}^{2}$ for skeletal muscle (Nigg and Herzog 1999), giving a maximally generated force of this muscle of $0.3 \mathrm{~N}$. An interesting comparison of the effect of this force on the malleus can be made by translating this number into a corresponding static pressure working on the TM from the ear canal side.
Dividing the force of $0.3 \mathrm{~N}$ by the (projected) area of the pars tensa of the TM of gerbil $2\left(13.64 \mathrm{~mm}^{2}\right.$, cf. Table 2), we obtain a (maximum) static pressure of $22 \mathrm{kPa}$. The magnitude of this pressure falls in the range of static pressures associated with scuba diving or taking an airplane.

The final merged 3D model shows that the tensor tympani muscle belly is larger than expected from visual observations. Its main part is hidden as it is situated in a gap between the spiraled cochlear dome and the bulla wall.

Stapedial artery. A typical anatomical feature of the gerbil ME is the stapedial artery running through a bony channel on the surface of the first cochlear turn and passing through the stapes crura in the ME air cavity. Using OPFOS, it was possible to image this relatively large stapedial artery, cf. Figure 9 . We could even distinguish and separately model the stapedial artery soft tissue wall (the actual blood vessel) and its fluid-filled lumen.

The diameter of the blood vessel was the smallest in between the crura and amounted to $355 \mu \mathrm{m}$ with (i.e., outer diameter) and $275 \mu \mathrm{m}$ without (i.e., inner diameter) the blood vessel soft tissue wall. The wall had a thickness of about $40 \mu \mathrm{m}$.

Stapedius muscle and tendon. After segmentation of the stapedius muscle and tendon, we end up with the mesh shown in Figure 9. The tiny volume enclosed in this (tendon and muscle) mesh amounts to $0.085 \mathrm{~mm}^{3}$, and the two most distant points on the combined structure are $1.81 \mathrm{~mm}$ apart. The diameter of the tendon varies between 40 and $55 \mu \mathrm{m}$. If we again divide the volume by an estimated average muscle fiber length of $350 \mu \mathrm{m}$, we get a cross-sectional area of $2.4 \times 10^{-3} \mathrm{~cm}^{2}$. Multiplying this value by $25 \mathrm{~N} / \mathrm{cm}^{2}$ gives an estimation of $0.06 \mathrm{~N}$ for the maximum force the muscle can produce.

TABLE 2

Geometry parameters of the TM for G1, G2, and G3 ear components, derived from the 3D surface meshes obtained from $\mu \mathrm{CT}$

\begin{tabular}{|c|c|c|c|}
\hline & $T M G 1$ & $T M G 2$ & $T M G 3$ \\
\hline Diameter tympanic ring inferior-superior ( $\mathrm{mm}$ ) (parallel to the direction of the manubrium) & 4.114 & 4.144 & 4.168 \\
\hline Diameter tympanic ring anterior-posterior ( $\mathrm{mm}$ ) (orthogonal to the direction of manubrium) & 4.190 & 4.051 & 4.169 \\
\hline Average diameter of the pars flaccida ring $(\mathrm{mm})$ & 1.492 & 1.335 & 1.413 \\
\hline Angle between manubrium and plane of tympanic ring $\left(^{\circ}\right)$ & 22.6 & 21.4 & 23.4 \\
\hline Distance between umbo and plane of tympanic ring $(\mathrm{mm})$ & 0.765 & 0.760 & 0.769 \\
\hline Surface area pars tensa $\left(\mathrm{mm}^{2}\right)$ & 17.96 & 17.50 & 17.35 \\
\hline Surface area pars flaccida $\left(\mathrm{mm}^{2}\right)$ & 2.46 & 2.24 & 2.11 \\
\hline Projected surface area pars tensa onto the plane of tympanic ring $\left(\mathrm{mm}^{2}\right)$ & 13.69 & 13.64 & 13.22 \\
\hline Projected surface area pars flaccida onto the plane of tympanic ring $\left(\mathrm{mm}^{2}\right)$ & 1.42 & 1.45 & 1.54 \\
\hline Thickness near annulus $(\mathrm{mm})^{\mathrm{a}}$ & \pm 0.035 & & \\
\hline Minimal thickness between annulus and umbo $(\mathrm{mm})^{\mathrm{a}}$ & \pm 0.007 & & \\
\hline Thickness near umbo $(\mathrm{mm})^{\mathrm{a}}$ & \pm 0.020 & & \\
\hline
\end{tabular}

G1 gerbil 1, G2 gerbil 2, G3 gerbil 3

${ }^{\mathrm{a}}$ Mean thickness data from confocal microscopy on 11 gerbils by Kuypers et al. (2005) 
TABLE 3

3D length of the manubrium (umbo tip till lateral process tip, cf. Fig. 5) and 3D height of the stapes (medial footplate till tip stapes head) for G1, G2, and G3, derived from the 3D surface meshes obtained from $\mu \mathrm{CT}$

\begin{tabular}{lc}
\hline & Distance $(\mathrm{mm})$ \\
\hline Manubrium G1 & 2.747 \\
Manubrium G2 & 2.704 \\
Manubrium G3 & 2.729 \\
Stapes footplate-head G1 & 1.277 \\
Stapes footplate-head G2 & 1.264 \\
Stapes footplate-head G3 & 1.273 \\
\hline
\end{tabular}

G1 gerbil 1, G2 gerbil 2, G3 gerbil 3

The merged 3D model shows that the stapedius muscle body is attached to the lateral (horizontal) semicircular canal, cf. Figure 9. In the figure, a gap is seen between the semi-circular canal and the muscle because only the fluid-filled cavity of the canal is shown. When showing bone as well, one sees the muscle clasps firmly around the lateral semi-circular canal wall.

Joint clefts. As can be seen in Figure 2B, the incudomallear and incudostapedial joints can be easily distinguished on high-resolution OPFOS cross sections and appear to form a tight connection. $\mu \mathrm{CT}$ data also show both clefts, from which we made 3D meshes.

The incudomallear joint connects the incus and malleus and has the shape of a twisted saddle. The gap or cleft between the ossicles could contain synovial fluid as it is considered a synovial joint; however, this is not confirmed from our OPFOS measurements nor $\mu \mathrm{CT}$ data in gerbil. No fluid or open space is detected in the joint cleft, and the joint seems quite rigid. This rigidness was already reported for other species by Guinan and Peake (1967) and Gundersen and Høgmoen (1976). The thickness of the joint varies from nearly 0 to $51 \mu \mathrm{m}$. The gap or joint tissue is thinner at the lateral side.

The incudostapedial joint connects the incus lenticular process with the head of the stapes. Our model of this synovial joint shows an oval disk with an

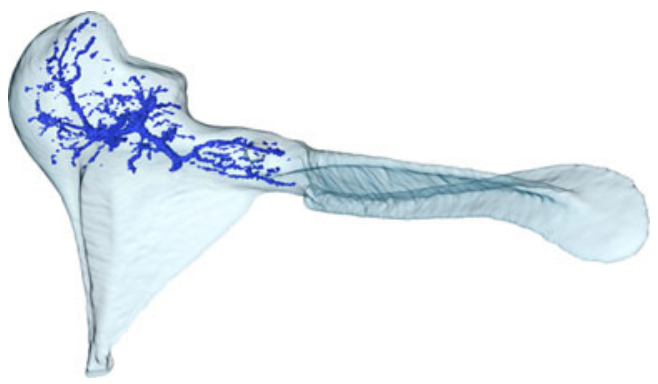

FIG. 5. Mesh of the malleus (gerbil 2) rendered transparent in combination with a mesh of the (major) blood vessel channels running inside it. Data obtained from $\mu$ CT. Voxel size is $8.5 \times 8.5 \times 8.5 \mu \mathrm{m}$. approximately even thickness of $25.5 \mu \mathrm{m}$. Again, the joint cleft seems to possess no synovial fluid and forms a rigid connection, which has also been reported in cat (Funnell et al. 2005).

OPFOS also visualized the stapedial annular ligament cleft in which the annular stapedial ligament is situated, forming a syndesmosis joint. A syndesmosis is a slightly movable articulation where bony surfaces are tightly united by a fibrous tissue ligament (Laurent 1998). The high resolution of the OPFOS data allows to make a 3D mesh of this thin structure, cf. Figure 10. The thickness of the ligament varies between 8 and $18 \mu \mathrm{m}$, confirmed by the gap seen in the $\mu \mathrm{CT}$ cleft model which is about $12-18 \mu \mathrm{m}$.

Chorda tympani. The chorda tympani nerve branches from the facial nerve and runs through the ME air cavity. In gerbil, the nerve jumps from a sort of support beam at the superior bulla wall to the malleus where it is tightly connected to the malleus neck in the vicinity of which the tensor tympani muscle connects as well, cf. Figure 7. It hangs in the ME air space passing the incudal long process laterally and the manubrium medially. It rounds the malleus neck from the posterior to the anterior side, passing the tensor tympani tendon inferiorly. At the anterior side, it lies on the anterior process sheet until it disappears in a fissure of the bulla wall again. It was unexpected that the chorda tympani could be visualized so well in OPFOS cross sections, cf. Figure 8 , because myelin nerve sheets can in principle not be made transparent by the Spalteholz process. Apparently, because the nerve is thin enough, the blurring effect of the less transparent chorda tympani was negligible.

\section{Merging of CT and OPFOS models}

As described before, we obtained a series of crosssectional images from $\mu \mathrm{CT}$ with bone only, cf. Figure 3, and from OPFOS with bony and soft tissue structures, $\mathrm{cf}$. Figures 2, 6, and 8. With OPFOS, we performed ROI recording of all soft tissue structures, so only incomplete parts of the ossicles were measured. However, using these partial models of ossicles and/or bulla bone that were recorded together with the soft tissue, we could align these bony structures (and thus the soft tissue structures as well) to the $\mu$ CT bone models, cf. Figure 11, using the Procrustes superimposition method.

The merging and alignment of bony structures revealed that some shrinking of the gerbil 2 specimen had occurred despite of our careful efforts during preparation. Using the warping procedure in Amira (similar to the Procrustes superimposition method, only allowing for scaling in every dimension as well), we found a shrinking factor of $8.4 \%$ in all three dimensions. After applying the spatial transformation and upscaling, the OPFOS soft tissue meshes fit rather 

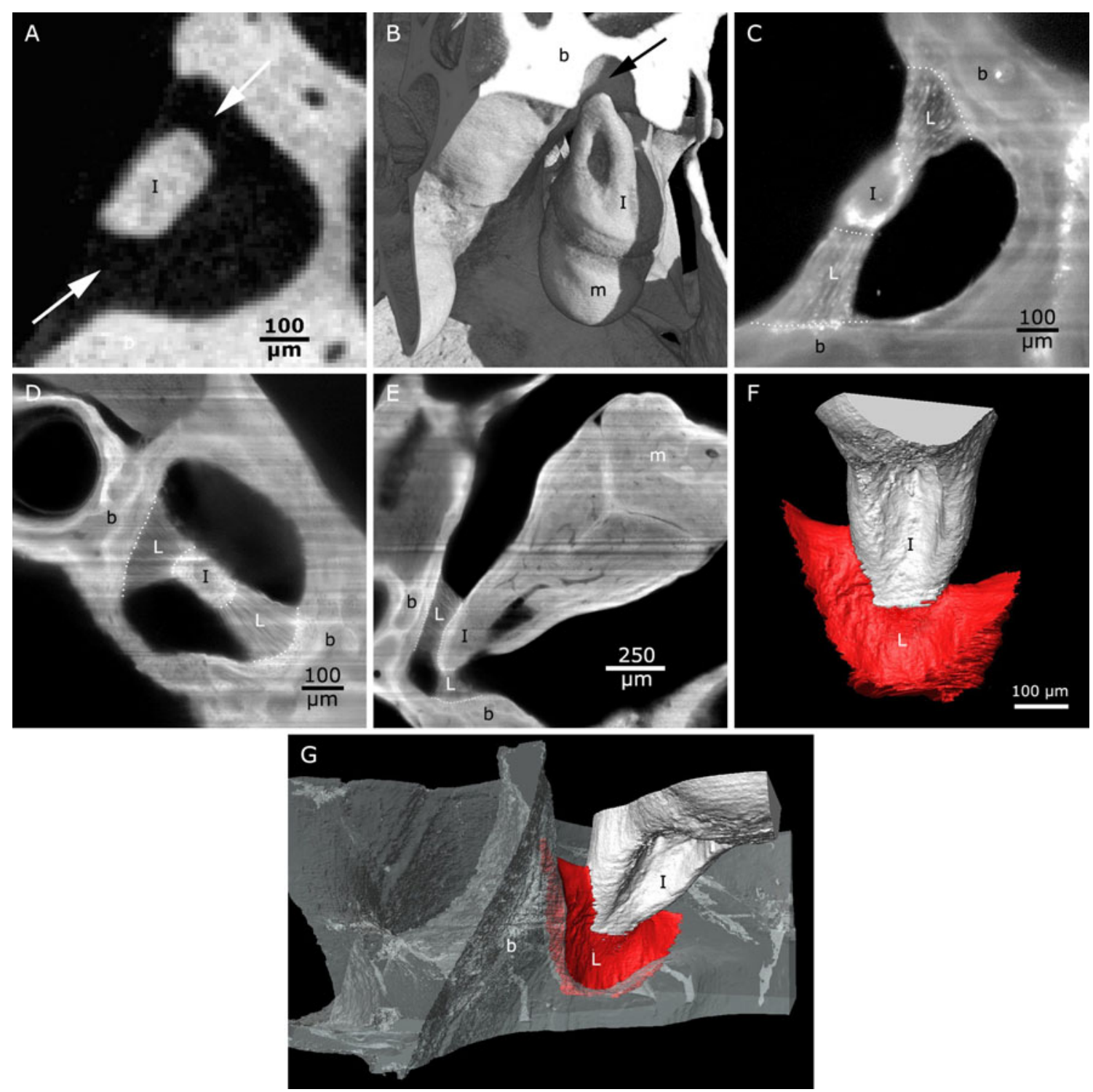

FIG. 6. A $\mu \mathrm{CT}$ cross section and $\mathbf{B} 3 \mathrm{D} \mu \mathrm{CT}$ reconstruction from automatic thresholding do not show the posterior incudal ligament in the bony wall recess. Arrows indicate the position of the invisible ligament. Pixel (and voxel) size is $8.5 \times 8.5(\times 8.5) \mu \mathrm{m}$. C-E ROI OPFOS cross sections from different orientations do show the ligament in the recess. F, G 3D OPFOS meshes. Voxel size is $0.97 \times 0.97 \times 2.5 \mu \mathrm{m}$. $b$ bulla, $l$ incus, $L$ ligament, $m$ malleus.

well in between the CT bone mesh models. For instance, corresponding bony parts of the malleus from OPFOS using a scale factor of $8.4 \%$ were aligned with the malleus from $\mu \mathrm{CT}$. After applying the same scaling and spatial transformation to the tensor tympani, its tendon attaches to the malleus, cf.
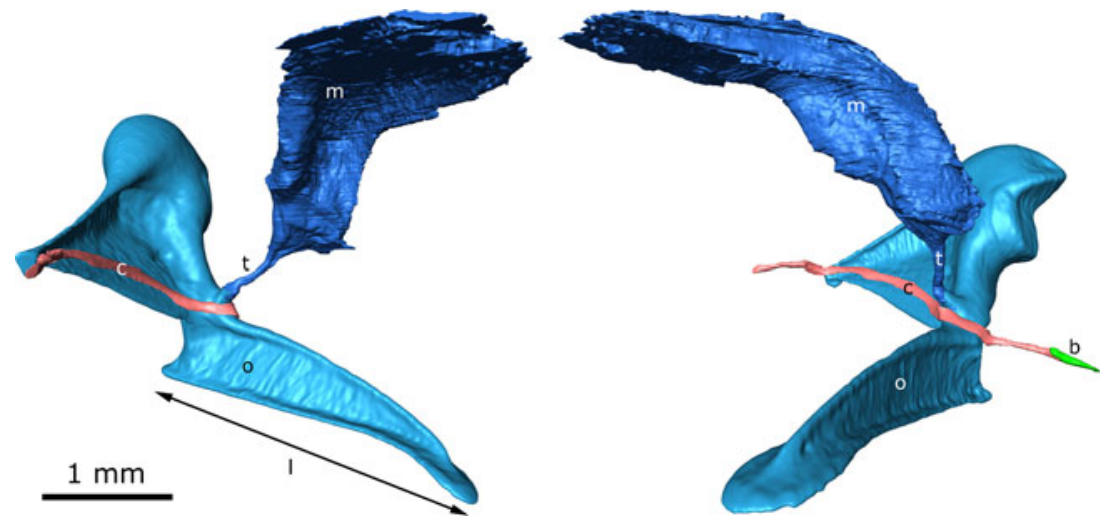

FIG. 7. Two views of the topography of the chorda tympani in combination with the malleus and the tensor tympani muscle and tendon (gerbil 2). The soft tissue data originate from OPFOS (voxel size is $2 \times 2 \times 4.5 \mu \mathrm{m}$ ), while the malleus data come from $\mu \mathrm{CT}$ (voxel size is $8.5 \times$ $8.5 \times 8.5 \mu \mathrm{m}) . b$ bulla, $c$ chorda tympani, $m$ muscle, $o$ malleus ossicle, $t$ tendon, $I$ manubrium length. 

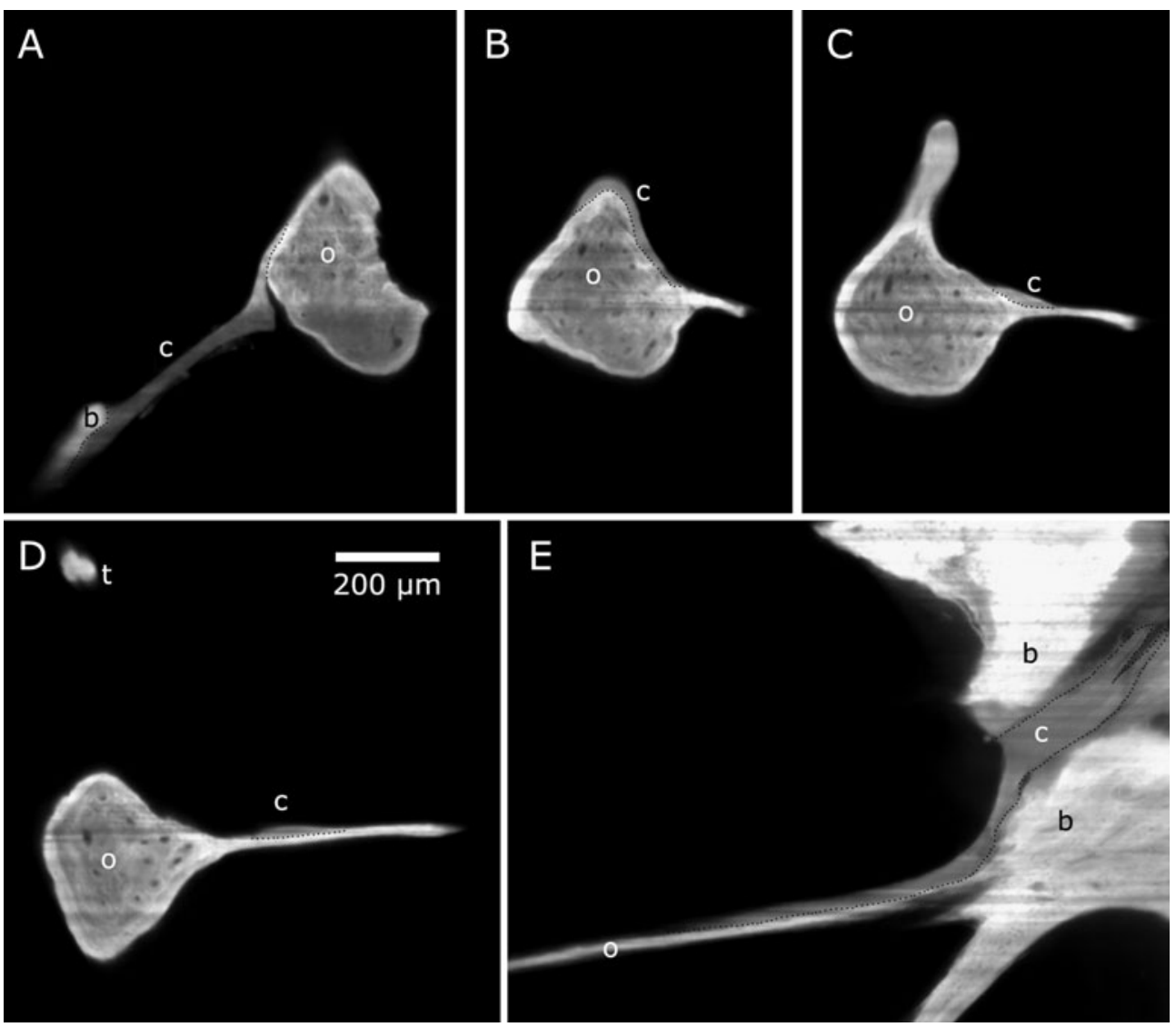

FIG. 8. OPFOS cross sections showing the course of the chorda tympani with respect to the malleus. A Chorda tympani jumps from a bony support beam to the malleus neck superior side. B It rounds the malleus neck below the tensor tympani. C-E It continues on the anterior process sheet until it enters a fissure in the bulla wall. $b$ bulla, $c$ chorda tympani, $o$ malleus ossicle, $t$ tendon. All subfigures are of the same scale.

Figure 11, and at the other side, its muscle body inserts nicely in a bony cavity of the bulla of the inverse shape, cf. Figure 12. This and similar facts give us confidence in the merging of the data.

\section{DISCUSSION}

\section{Imaging method}

Several methods exist to measure and image the ME for the creation of FEM models. $\mu \mathrm{CT}$ in itself is mainly suited to image the bony structures. $\mu \mathrm{CT}$ using contrast agents is a valuable alternative to our combined approach (Metscher 2009). However, it is difficult to discriminate between bone and soft tissue, so it would be necessary to do $\mu \mathrm{CT}$ scans before and after staining and merge the data as we now did with OPFOS. OPFOS offers a resolution down to $2 \mu \mathrm{m}$, which is seldom achieved in $\mu \mathrm{CT}$. For this reason, we preferred OPFOS to obtain the soft tissue data. Multiple energy CT techniques have also proven to be a valuable method for discriminating between soft tissue and bone in CT images (Johnson et al. 2007; Granton et al. 2008). For large macroscopic structures, the technique is indeed feasible; however, it becomes more difficult in the case of microscopic samples. The position of the micro-focus spot changes in an X-ray tube when its energy or source is altered. As a result, the datasets are slightly shifted in a complicated way, and tissue discrimination can no longer be done by simple subtraction or division. Gradually, these technical issues are being solved, so in the future dual-energy CT may be used to measure and discriminate soft tissue and bone.

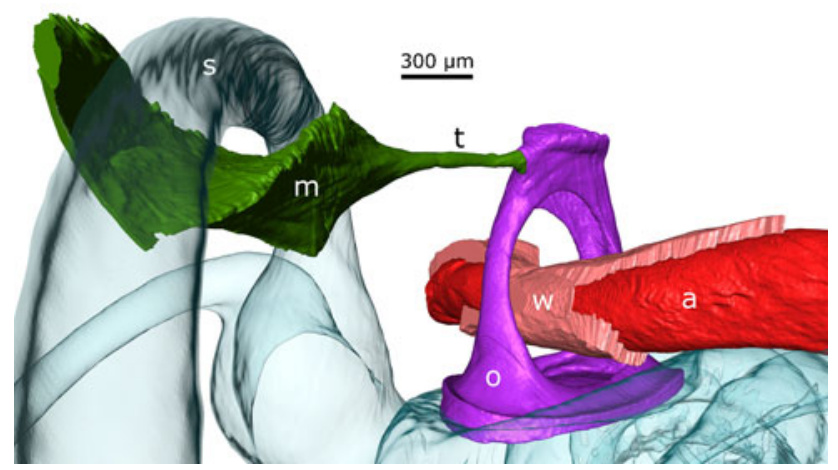

FIG. 9. Stapes bone, stapedius muscle and tendon, and stapedial artery models obtained from OPFOS (voxel size is $1.5 \times 1.5 \times 5 \mu \mathrm{m}$ ) and the fluid-filled cavity of the horizontal semi-circular canal from $\mu \mathrm{CT}$ (voxel size is $8.5 \times 8.5 \times 8.5 \mu \mathrm{m}$ ) are shown (gerbil 2 ). a artery, $m$ muscle, $o$ stapes ossicle, $s$ semi-circular canal, $t$ tendon, $w$ artery wall. 
A
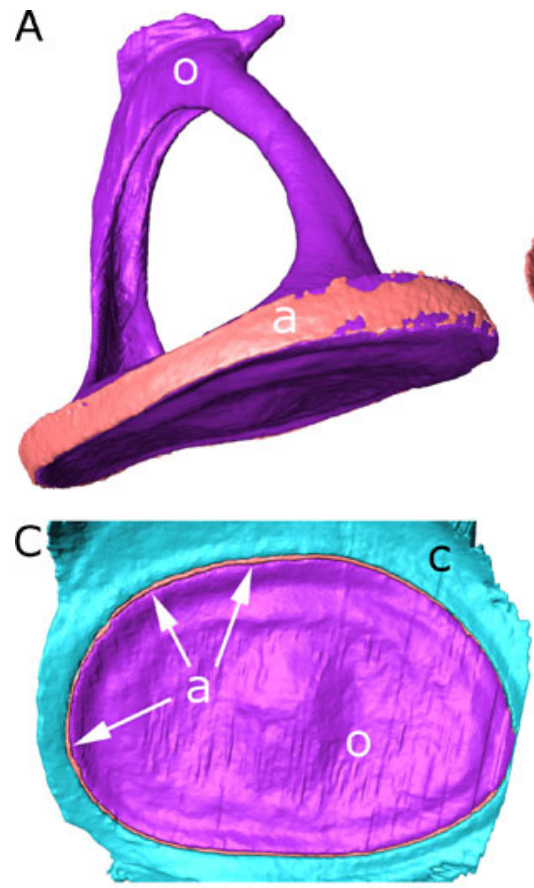

E

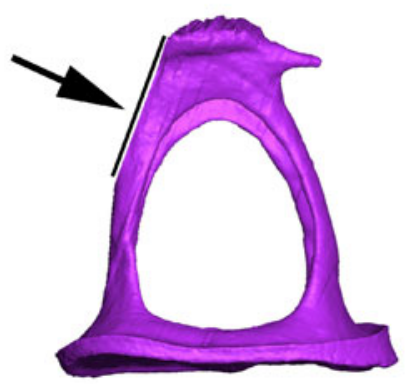

B
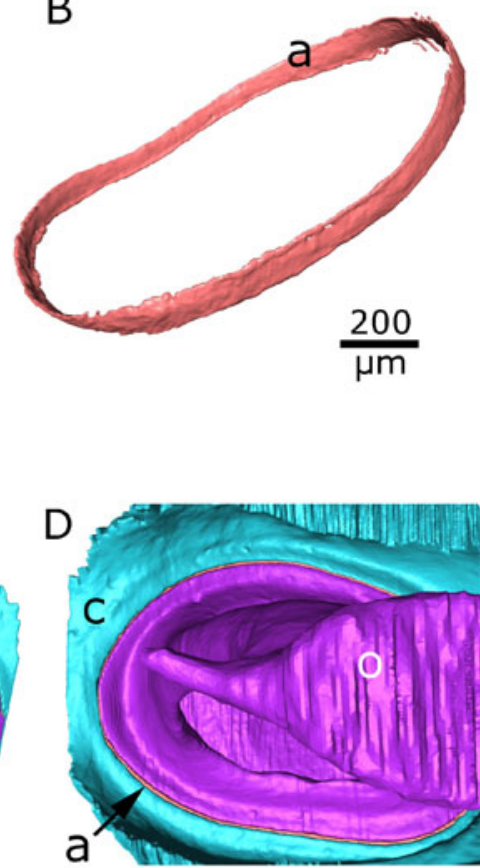

$\mathrm{F}$

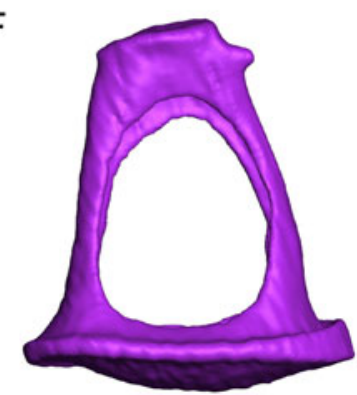

FIG. 10. A-E OPFOS-based models of the stapes and the stapedial annular ligament (gerbil 2). F $\mu$ CT-based model of the stapes (gerbil 2). The footplate modeled from $\mu \mathrm{CT}$ data is convex, while in the OPFOS model it is not. a annular ligament, $c$ cochlea, $o$ stapes ossicle. The arrow indicates the end of the OPFOS dataset.
The most used alternative to our method is conventional histological sectioning, which is unsurpassed in resolution and produces data on the bone and soft tissue simultaneously. Both the histological method and our combined method need a similar specimen preparation that can induce shrinking (Lane and Ráliš 1983; Henson et al. 1994). Our method is considered non-destructive (as multiple measurements can be done on the sample) while the histological method can only measure the sample once and in one slicing orientation because of the need for physical cutting of the specimen. Furthermore, these 2D slices are often deformed during slicing, requiring difficult image processing and registration of all slices before generating a 3D model. $\mu \mathrm{CT}$ and OPFOS each deliver perfectly and automatically aligned cross sections that require no postprocessing. Instead of registering every 2D slice, our method only needs to register complete 3D meshes of all submodels to one another. OPFOS further allows real-time virtual sectioning and imaging.

The OPFOS method is one of the the first techniques in a growing field of (laser) light-sheet, now known as the (laser) light-sheet-based fluorescence microscopy
(LSFM). The many different implementations and improvements of the technique have been listed in a review article by Buytaert et al. (2011). The construction of an OPFOS/LSFM setup is well feasible in the sense that all parts needed are readily available on the market. Researchers interested in the construction of such a setup or in the collaboration are welcome to contact the authors, and even the first commercial devices are becoming available (Buytaert et al. 2011).

\section{Human versus gerbil}

When using animal models, it is important to be aware of the differences with human ME morphology.

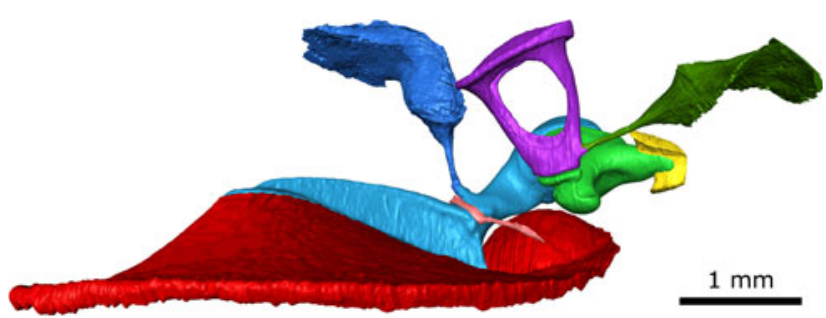

FIG. 11. Merged OPFOS-CT ME model (gerbil 2). 


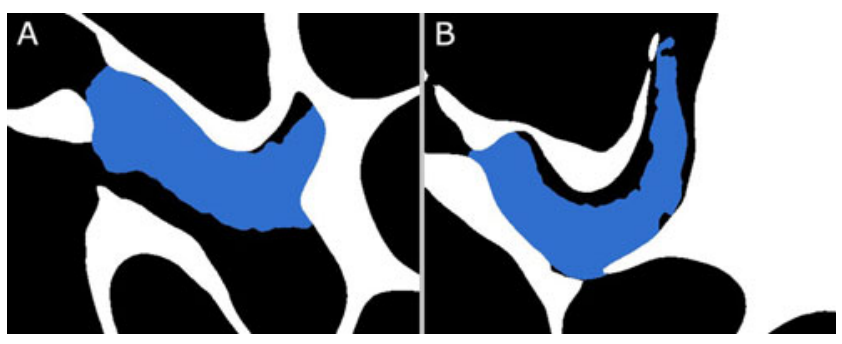

FIG. 12. Cross sections at different depths through the 3D merged models of the bulla bone (white) from $\mu \mathrm{CT}$ and the tensor tympani (blue) from OPFOS. Black represents air-filled space such as the ME air cavity. The tensor tympani fits nicely in the bone, rather touching the cavity wall than overlapping with it.

Figure 13 shows a schematic representation of all human ME components. In addition to the data prepared in this paper, we confirmed our findings in other gerbil ears during other studies using OPFOS and visual inspection with the operation microscope.

We found that in gerbil, no superior incudal, no superior mallear, and no lateral mallear ligament are present, contrary to the case in humans. The presence and/or function of superior attachments to malleus and incus as suspensory structures is of controversy, though many mathematical models or drawings of the human ME include such structures, cf. Table 2.1 in Mikhael (2005) and Merchant and Nadol (2010).

It has been proposed by Rosowski et al. (1999) that the anterior mallear ligament is a bony connection to the bulla, while Elkhouri et al. (2006) observed the presence of some connective tissue. Our OPFOS measurements could not distinguish any soft tissue, and our CT measurement showed an ossified or cartilaginous connection. The anterior process also had a less pronounced shape in human than the Japanese fan-shaped structure in gerbil.

The posterior incudal ligament, which connects the incus short crus to the fossa incudis, exists in many different configurations, as is illustrated in Figure 14 by Funnell (1972) (based on work by Kobayashi). From the OPFOS sections, cf. Figure 6C-E, the gerbil posterior ligament appears to fall in the category of human and cat configurations. However, it is only possible to appreciate the true configuration in $3 \mathrm{D}$, cf. Figure 6F, G, which clearly places this gerbil ligament in the category of guinea pig and rabbit. The posterior incudal ligament consists of one sickleshaped part. According to Sim and Puria (2008), it has been observed that in human, the two parts shown in Figure 14 are also connected around the tip of the short crus of the incus to form a single continuous ligament rather than two separate ligaments. In this respect, gerbil and human ME then would be alike.

We also found the chorda tympani nerve to be present in a special arrangement in gerbil and more tightly connected to the malleus ossicle than in human: In human, this nerve traverses the open space of the ME cavity without actually attaching to the ossicles. In gerbil, there exists a tight connection with the malleus neck and the nerve lies on top of the Japanese fan-shaped anterior process sheet, cf. Figures 7 and 8. Furthermore, the topographic relation of the chorda tympani to the tensor tympani muscle differs from human. In gerbil, it runs hypotensoric (inferiorly to the tensor tympani) and in between the muscle and manubrium, as was confirmed in a recent publication by Ruf et al. (2009), while in human it passes epitensoric (superiorly to the tensor tympani), e.g., Maier (2008).

We derived the ossicle bone density from our volume measurements and from mass data from the literature. We obtained an ossicle bone density of $1.37 \times 10^{3} \mathrm{~kg} / \mathrm{m}^{3}$ for the stapes and $1.74 \times 10^{3} \mathrm{~kg} / \mathrm{m}^{3}$ for incus and malleus. In comparison to human, the averaged malleus density is found to be $2.31 \times 10^{3} \mathrm{~kg} /$ $\mathrm{m}^{3}$, and the averaged incus density is $2.14 \times 10^{3} \mathrm{~kg} / \mathrm{m}^{3}$ (Sim and Puria 2008). Another source mentions an average stapes density of $2.2 \times 10^{3} \mathrm{~kg} / \mathrm{m}^{3}$ in human (Kirikae 1960; Gan et al. 2004). Hence, gerbil ossicle densities appear to be significantly lower than in human.
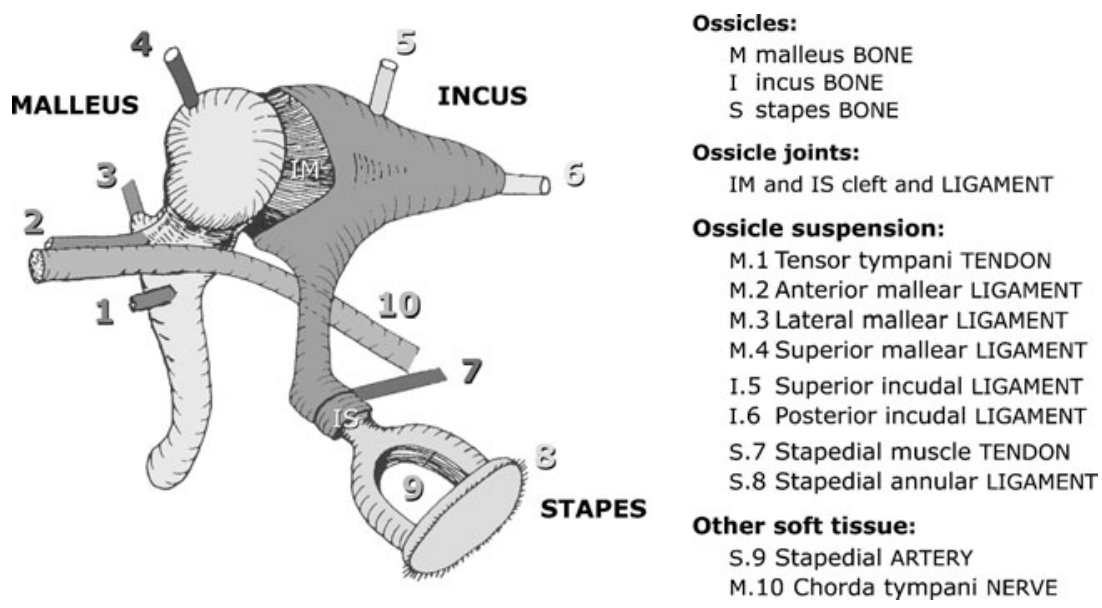

FIG. 13. General schematic overview of all relevant middle ear components in human. 


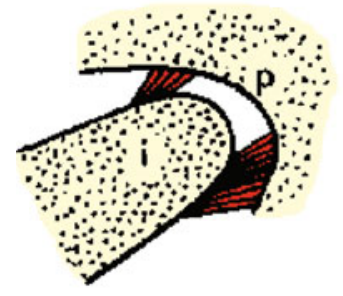

human, cat

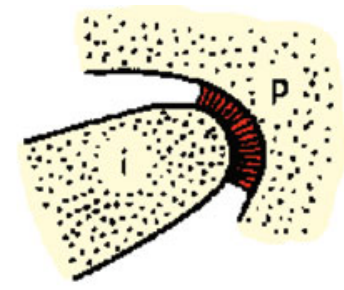

guinea pig, rabbit

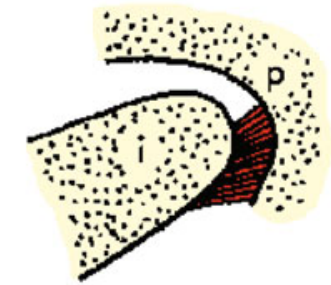

pig, fox

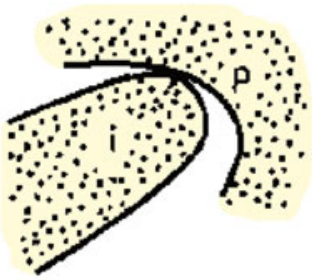

horse, goat

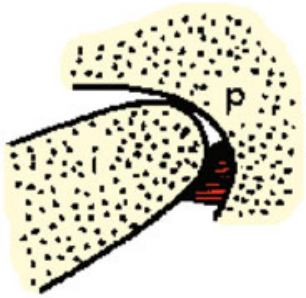

cow, sheep
FIG. 14. Schematic representation of different posterior incudal ligament configurations per species (courtesy of Funnell 1972). Gerbil falls in the category of guinea pig and rabbit.
Another contrast to human is that the stapedial artery is usually present in gerbil, while seldom in human. Finally, our observations show that the gerbil manubrium of malleus is tightly fused over its full length with the TM, while in human it is mainly only fixed at the tip and lateral process of the manubrium (Koike et al. 2002).

\section{Resolution}

We used state-of-the-art X-ray micro-computed tomography and the relatively new orthogonal-plane fluorescence optical-sectioning microscopy on the ME. In previous CT-based studies of the ME, the following model resolutions were reported: $5.5 \mu \mathrm{m}$ on gerbil (Elkhouri et al. 2006), $6 \mu \mathrm{m}$ on human (Hagr et al. 2004), $10 \mu \mathrm{m}$ on cat (Decraemer et al. 2003), and $10 \mu \mathrm{m}$ on human (Vogel 1999). Though these numbers are comparable to our isometric $8.5-\mu \mathrm{m}$ voxel size for $\mu \mathrm{CT}$ on gerbil bone, our data and models are of much higher quality than those shown in previous work. One reason might be that the previous authors stated voxel size instead of resolution, while we actually achieve a true resolution of $8.5 \mu \mathrm{m}$. Other factors such as scan parameter settings could also account for differences in image quality.

ME soft tissue imaged with medical CT devices gave poor resolution (Lemmerling et al. 1997), and $\mu \mathrm{CT}$ delivered modest resolution (Sim and Puria 2008). The same goes for MRI measurements of gerbil soft tissue structures, e.g., $45 \mu \mathrm{m}$ (Elkhouri et al. 2006). OPFOS is clearly better suited to achieve high-resolution sections on ligaments and muscles - with pixel sizes ranging from 1 to $5 \mu \mathrm{m}$-as can be seen from our sections and 3D models, cf. Figures 2, 6, 7, 8, 9, 10, and 11.

\section{Artifacts}

Segmentation of the fluid-filled inner ear channels in the $\mu \mathrm{CT}$ data showed that the round window in all three models is prominently bulged inward toward the cochlea. This might indicate either a small overpressure in the ME air cavity or a loss of cochlear fluid because of dehydration or leakage.

Merging of OPFOS and $\mu \mathrm{CT}$ data revealed shrinking of the soft and bony tissue, most likely caused by the elaborate OPFOS specimen preparation (e.g., tissue fixation, decalcification, dehydration, and Spalteholz treatment), though previous authors reported that this procedure induced negligible shrinking (Voie 2002; Valk et al. 2005; Hofman et al. 2008). Thanks to the combination of OPFOS with $\mu \mathrm{CT}$, we have undeformed reference data that we can use to derive a scaling factor. Homogeneous scaling with $8 \%$ of the OPFOS (bone and soft tissue) models has partially corrected for the shrinking artifact. After decalcification of the sample, bone is reduced to a collagen matrix. The effect of decalcification cannot be investigated with $\mu \mathrm{CT}$ as all calcium is removed and X-ray absorption becomes negligible. It is, however, a reasonable assumption that dehydration will have a similar (and homogeneous) shrinking effect on both soft tissue and decalcified bone. In histology, the same specimen preparation (decalcification and dehydration) is performed, and the same assumption (homogeneous shrinkage) is adopted.

Another artifact related to specimen preparation was noticed on the stapes. The footplate of the stapes is clearly convex and bulges inward to the cochlea in the $\mu \mathrm{CT}$ models, e.g., Figure 10. After decalcification, dehydration, and Spalteholz treatment, the footplate of the OPFOS model showed some relaxation and shriveling of its convex shape. The models available 
for download therefore consist of $\mu \mathrm{CT}$ data for bone and OPFOS data for soft tissue meshes.

Stripe artifacts in OPFOS were strongly reduced but not entirely eliminated by our bi-directional illumination/sectioning OPFOS setup. In combination with manual segmentation, which also partially corrects for this artifact, no effect remained in the models so no image post-processing of the data was required.

OPFOS was not suited to image the TM, but the full-field outline of the TM shape was obtained from $\mu \mathrm{CT}$ : We could not measure a volume model of the TM with the correct thickness, but only a surface model. FEM modelers can, however, use the surface shape directly as a shell model, cf. Gan et al. (2004) and Elkhouri et al. (2006), and apply either a uniform or a varying measured thickness distribution to their own choosing (as different approaches are taken by different modelers). Table 2 mentions average thickness data at three TM regions, measured on 11 gerbil TMs with confocal microscopy (Kuypers et al. 2005).

\section{Open-source availability}

All 3D data and surface mesh models presented in this paper are freely available for educational and research purposes on the website of the Laboratory of BioMedical Physics (http://www.ua.ac.be/bimef/models).

Several educational and research 3D models have also been made available in the past ("3D Virtual Models of the Human Temporal Bone and Related Structures" of Eaton-Peabody Laboratory of Auditory Physiology, http://research.meei.harvard.edu/Otopathology/ 3dmodels/download.html; "3D Overview of Ear Anatomy" of Ear \& Auditory Research Laboratory, http: / / earlab.medicine.dal.ca/3D/3D-Overview.htm; "3D Ear Human Ear" of Auditory Mechanics Laboratory, http:/ / audilab.bmed.mcgill.ca/ daren/3Dear/; "The Vertebrate Ear and Temporal Bone" of Auditory Research Laboratory, http:/ / cbaweb2.med.unc.edu/ henson mrm/; "MicroCT Data and 3D Reconstructions" of OtoBiomechanics Group, http://www.stanford.edu/ $\sim$ purial/Site/Imaging.html).

\section{CONCLUSION}

Finite-element computer modeling needs accurate 3D models to obtain realistic simulation results for middle ear mechanics. 3D models are also useful in medical training or for the interpretation and presentation of experimental results. The middle ear does not only comprise the ossicles but also consists of soft tissue: tympanic membrane, ligaments, muscles, tendon, and blood vessels.
In this paper, we presented an accurate and complete morphological 3D middle (and inner) ear model of gerbil. The model is freely available to the research community at our website. The presented model quality is unprecedented. The position, orientation, and size of all components making up the gerbil middle ear are now accurately known and individually discussed.

\section{ACKNOWLEDGMENTS}

We gratefully acknowledge the financial support of the Research Foundation-Flanders and the Fondation Belge de la Vocation. We thank Pieter Vanderniepen for his assistance in operating the CT device, Magnus Von Unge and Wim Decraemer for their feedback on human and gerbil anatomy, Fred Wiese for manufacturing the vial holder, Robert Funnell for the use of Figure 14, and Peter Aerts for the feedback on muscle functionality.

\section{REFERENCES}

Beer H, Bornitz M, Hardtke H, Schmidt R, Hofmann G, Vogel U, ZAHNert T, HuttenBrink K (2000) Modelling of components of the human middle ear and simulation of their dynamic behaviour. Audiology Neurotology 4:156-162

Blayney A, Williams KR, Rice HJ (1997) A dynamic and harmonic damped finite element analysis model of stapedotomy. Acta Otolaryngol 117:269-273

BUytaerT JAN (2010) New optical tomographic \& topographic techniques for biomedical applications. University Press Zelzate, Zelzate

Buytaert JAN, Aernouts J, Dirckx JIJ (2009) Indentation measurements on the eardrum with automated projection moiré profilometry. Optics Lasers Engineering 47:301-309

Buytaert JAN, Descamps E, Adriaens D, Dirckx JJJ (2011) The OPFOS microscopy family: high-resolution optical-sectioning of biomedical specimens. Anat Res Int. http://arxiv.org/abs/ 1106.3162

Buytaert JAN, Dirckx JJJ (2007) Design and quantitative resolution measurements of an optical virtual sectioning three-dimensional imaging technique for biomedical specimens, featuring two-micrometer slicing resolution. J Biomed Opt 12:014039

BuYTAERT JAN, DirckX JIJ (2009) Tomographic imaging of macroscopic biomedical objects in high resolution and three dimensions using orthogonal-plane fluorescence optical sectioning. Appl Opt 48:941-948

Cheng T, GAN RZ (2008) Experimental measurement and modeling analysis on mechanical properties of tensor tympani tendon. Medical engineering Physics 30:358-366

Decraemer WF, Dirckx JJJ, FunNell WRJ (2003) Three-dimensional modelling of the middle-ear ossicular chain using a commercial high-resolution X-ray CT scanner. J Assoc Res Otolaryngol: JARO $4: 250-263$

Decraemer WF, Khanna SM, Dirckx IJJ (2002) The integration of detailed 3-dimensional anatomical data for the quantitative description of 3-dimensional vibration of a biological structure. An illustration from the middle ear. In: Tomassini EP (ed) Fifth international conference on vibration measurements by laser 
techniques: advances and applications. SPIE, Bellingham, pp $148-158$

Dierick M, Masschaele B, Hoorebeke LV (2004) Octopus, a fast and user-friendly tomographic reconstruction package developed in LabView $^{\circledR}$. Meas Sci Technol 15:1366-1370

Eiber A, Freitag HG, Burkhardt C, Hemmert W, Maassen M, Rodriguez Jorge J, Zenner HP (2000) Dynamics of middle ear prosthesessimulations and measurements. Audiology Neurotology 4:178-184

Elkhouri N, Liu H, Funnell WRJ (2006) Low-frequency finiteelement modeling of the gerbil middle ear. J Assoc Res Otolaryngol: JARO 7:399-411

FunNeLl WRJ (1972) The acoustical impedance of the guinea-pig middle ear and the effects of the middle-ear muscles. J Acoust Soc Am 52:129

Funnell WrJ, Heng Siah T, McKee MD, Daniel SJ, Decraemer WF (2005) On the coupling between the incus and the stapes in the cat. J Assoc Res Otolaryngol: JARO 6:9-18

FunNell WRJ, Khanna SM, DeCRAEMER WF (1992) On the degree of rigidity of the manubrium in a finite-element model of the cat eardrum. J Acoust Soc Am 91:2082-2090

FunNeLl WRJ, LaszLo CA (1978) Modeling of the cat eardrum as a thin shell using the finite-element method. J Acoust Soc Am 63:1461-1467

Gan RZ, Feng B, Sun Q (2004) Three-dimensional finite element modeling of human ear for sound transmission. Ann Biomed Eng 32:847-859

Gan RZ, Reeves BP, Wang X (2007) Modeling of sound transmission from ear canal to cochlea. Ann Biomed Eng 35:2180-2195

Gea SLR, Decraemer WF, Dirckx JJJ (2005) Region of interest microCT of the middle ear: a practical approach. J Xray Sci Technol 13:137-148

Granton PV, Pollmann SI, Ford NL, Drangova M, Holdsworth DW (2008) Implementation of dual- and triple-energy cone-beam micro-CT for postreconstruction material decomposition. Medical Physics 35:5030

Guinan J, Peake WT (1967) Middle-ear characteristics of anesthetized cats. J Acoust Soc Am 41:1237-1261

Gundersen T, Høgmoen K (1976) Holographic vibration analysis of the ossicular chain. Acta Otolaryngol 81:16-25

Hagr AA, Funnell WRJ, Zeitouni AG, Rappaport JM (2004) Highresolution X-ray computed tomographic scanning of the human stapes footplate. J Otolaryngol 33:217-221

Henson MM, Henson OW, Gewalt SL, Wilson JL, Johnson GA (1994) Imaging the cochlea by magnetic resonance microscopy. Hear Res 75:75-80

Hofman R, Segenhout JM, Buytaert JAN, Dirckx IJJ, Wit HP (2008) Morphology and function of Bast's valve: additional insight in its functioning using 3D-reconstruction. Eur Arch Otorhinolaryngol 265:153-157

Johnson TrC, Krauss B, Sedlmair M, Grasruck M, Bruder H, Morhard D, Fink C, Weckbach S, Lenhard M, Schmidt B, Flohr T, ReISER MF, BECKer CR (2007) Material differentiation by dual energy CT: initial experience. Eur Radiol 17:1510-1517

KIRIKAE I (1960) The structure and function of the middle ear. University of Tokyo Press, Tokyo

Koike T, Wada H, Kobayashi T (2002) Modeling of the human middle ear using the finite-element method. J Acoust Soc Am 111:1306-1317

Kuypers LC, Dirckx JJJ, Decraemer WF, Timmermans J-P (2005) Thickness of the gerbil tympanic membrane measured with confocal microscopy. Hear Res 209:42-52

LADAK HM, Funnell WRJ (1996) Finite-element modeling of the normal and surgically repaired cat middle ear. J Acoust Soc Am 100:933

LANE J, RÁLIš Z (1983) Changes in dimensions of large cancellous bone specimens during histological preparation as measured on slabs from human femoral heads. Calcif Tissue Int 35:1-4
LAURENT C (1998) The action of hyaluronan on repair processes in the middle ear. In: Laurent $\mathrm{C}$ (ed) The chemistry, biology and medical applications of hyaluronan and its derivatives. Portland, London, pp 283-289

Lee C-F, Chen P-R, Lee W-J, Chen J-H, Liu T-C (2006) Computer aided three-dimensional reconstruction and modeling of middle ear biomechanics by high-resolution computed tomography and finite element analysis. Biomed Engineering: Appl, Basis Commun $18: 214$

Lee DH, Chan S, Salisbury C, Kim N, Salisbury K, Puria S, BLEvins NH (2010) Reconstruction and exploration of virtual middle-ear models derived from micro-CT datasets. Hear Res 263:198-203

Lemmerling MM, Stambuk He, Mancuso AA, Antonelli PJ, Kubilis PS (1997) CT of the normal suspensory ligaments of the ossicles in the middle ear. AJNR Am J Neuroradiol 18:471-477

Lord RM, Abel EW, Mills RP (1999) A finite element model of the middle ear. In: Proceedings of NAFEMS WORLD CONGRESS '99 on effective engineering analysis, pp 487-494

MAIER W (2008) Epitensoric position of the chorda tympani in Anthropoidea: a new synapomorphic character, with remarks on the fissura Glaseri in Primates. In: Sargis EJ, Dagosto M (eds) Mammalian evolutionary morphology. Springer, Dordrecht, pp $347-360$

Masschaele B, Cnudde V, Dierick M, Jacobs P, Vanhoorebeke L, Vlassenbroeck J (2007) UGCT: New X-ray radiography and tomography facility. Nuclear Instruments and Methods in Physics Research Section A: Accelerators, Spectrometers, Detectors and Associated Equipment 580:266-269

Merchant SN, Nadol JB JR (2010) Schucknect's pathology of the ear. PMPH-USA, Shelton

Metscher BD (2009) MicroCT for comparative morphology: simple staining methods allow high-contrast 3D imaging of diverse nonmineralized animal tissues. BMC Physiol 9:1-14

Mikhael CS (2005) Finite element model of the human middle ear. Thesis, McGill University, Montréal, p 120

Mikhael CS, Funnell WRJ, Bance M (2004) Middle-ear finiteelement modelling with realistic geometry and a priori material-property estimates. In: 28th Ann Conf Can Med Biol Eng Soc, pp 126-129

Nigg BM, Herzog W (1999) Biomechanics of the musculo-skeletal system, 2nd edn. Wiley, New York

Nummela S (1995) Scaling of the mammalian middle ear. Hear Res $85: 18-30$

Prendergast P, Ferris P, Rice HJ, Blayney A (2000) Vibro-acoustic modelling of the outer and middle ear using the finite-element method. Audiology Neurotology 4:185-191

Puria S, Steele CR (2010) Tympanic-membrane and malleus-incuscomplex co-adaptations for high-frequency hearing in mammals. Hear Res 263:183-190

Rodt T, Ratiu P, Becker H, Bartling S, Kacher D, Anderson M, Jolesz F, KIKINIS R (2002) 3D visualisation of the middle ear and adjacent structures using reconstructed multi-slice CT datasets, correlating 3D images and virtual endoscopy to the 2D crosssectional images. Neuroradiology 44:783-790

Rosowski JJ, Ravicz ME, Teoh SW, Flandermeyer D (1999) Measurements of middle-ear function in the Mongolian gerbil, a specialized mammalian ear. Audiol Neurootol 4:129-136

Ruf I, Frahnert S, Maier W (2009) The chorda tympani and its significance for rodent phylogeny. Mammalian Biology-Zeitschrift fur Saugetierkunde 74:100-113

Sim JH, PURIa S (2008) Soft tissue morphometry of the malleusincus complex from micro-CT imaging. J Assoc Res Otolaryngol: JARO 9:5-21

Sun Q, Gan RZ, Chang K-H, Dormer KJ (2002) Computer-integrated finite element modeling of human middle ear. Biomech Model Mechanobiol 1:109-122 
Valk WL, Wit HP, Segenhout JM, Dijk F, van der Want JJL, Albers FWJ (2005) Morphology of the endolymphatic sac in the guinea pig after an acute endolymphatic hydrops. Hear Res 202:180-187

Vogel U (1999) New approach for 3D imaging and geometry modeling of the human inner ear. ORL 61:259-267

VoIE AH (2002) Imaging the intact guinea pig tympanic bulla by orthogonal-plane fluorescence optical sectioning microscopy. Hear Res 171:119-128
Voie AH, Burns DH, Spelman FA (1993) Orthogonal-plane fluorescence optical sectioning: three-dimensional imaging of macroscopic biological specimens. J Microsc 170:229-236

Wada H, Metoki T, KobaYashi T (1992) Analysis of dynamic behavior of human middle ear using a finite-element method. J Acoust Soc Am 92:3157-3168

Wang H, Northrop C, Burgess B, Liberman MC, Merchant SN (2006) Three-dimensional virtual model of the human temporal bone: a stand-alone, downloadable teaching tool. Otol Neurotol 27:452 